\title{
Echo Cancellation with Reference Signal Generator and Reliable Receiving Schemes for Intersymbol Interference
}

\author{
Hsiang-Feng Chi and Ja-Ling Wu
}

Department of Computer Science and Information Engineering, National Taiwan University, Taipei, 10764, Taiwan, Rep. of China

\begin{abstract}
In the adaptive echo cancellation (EC) of the full-duplex twowire data transmission, the problem of double-talk which disturbs adaptation procedure always exists since the far-end signal appeared continuously. To provide the reliable adaptation, the EC with decision-directed reference signal generator (RSG) was proposed.

Additionally, the intersymbol interference (ISI) is another problem. Fortunately, for EC with far-end RSG, the information of pulse shape dispersion in channel is included in the RSG and is helpful for reducing the effect of dispersive channel. In this paper, some methods which sufficiently make use of this information are proposed to provide optimal receiving and decoding, and hence a lower bit error rate is obtained.
\end{abstract}

\section{Introduction}

In the adaptive EC applications, the appearance of doubletalk will result in the interference and thus degrade the performance of adaptation. The double-talk detectors are commonly used to disable or slow down the adaptation when the interference is large. It may work in speech EC because the speech is bursty. However, in full-duplex two-wire data transmission, the far-end signal may continuously appear because the transmission is bidirectional. In other words, double-talk problem always exists in this situation. Fortunately, the pulse shapes of transmitted signal are of finite kinds. Hence the decision-directed scheme can be used to produce the far-end reference signal and eliminate the corresponding interference. The far-end pulse shapes are estimated adaptively and trifgered by estimated far-end data symbols so as generate: the counterpart of received far-end sigital (see [1]).

Due to the dispersion in the transmission, the pulse shape.s of far-end signal in the receiving-end are dispersed, and hence ISI will be resulted. Conventionally, this problem was considered and solved without any connection with the schernes of EC. However, for EC with far-end RSG, the information of dispersion in channel is included in the far-end reference signal estimator (RSE). Hence, this estimate seems helpful in dealing with the problem of ISI. With this motivation, we propose some methods which sufficiently make use of the information in far-end RSE to reduce the effect of dispersion in channel on the detection of far-end data symbols and thus gives a lower bit error rate.

In this paper, section 2 briefly describes the EC with RSG. In section 3 , the methods for reliable transmission using the information of far-end RSE will be proposed. In section 4 some computer simulations will be performed. Section 5 presents the conclusion of this paper.

\section{EC in Data Transmission with far-end RSG}

2.1 Models of the Transmission System and the Echo Cancellation

The digital subscriber loop provides a full-duplex two-wire data transmission in the telephone network, and is an important element of ISDN. However, there are always echo and channel dispersion problems in the transmission. The model of transmission and echo cancellation is discussed in the following, and the corresponding block diagram is shown in Figure 1.

Let $\{a(w)\}$ and $\{b(n)\}$ be the sequences of far-end and near-end data symbols, respectively. The sending signals in the far-end and near-end are respectively given by

$$
w(t)=\sum_{n=-\infty}^{\infty} a(n) s(t-n T), v(t)=\sum_{n=-\infty}^{\infty} b(n) s(t-n T) .
$$

where $s(t)$ is the transmitted pulse shape and $T$ is the baud interval.

Then, the received signal $r(t)$ can be written as

$$
r(t)=\sum_{n=-\infty}^{\infty} b(n) l(t-n T)+\sum_{n=-\infty}^{\infty} b(n) h(t-n T)+\nu(t)
$$

where $\{\nu(t)\}$ is a white Gaussian noise, and $l(t)=s(t) *$ $h(1), h(t)=s(t) * f(t) . h(t)$ is the impulse response of the transmission clannel, $f(t)$ is the impulse response of echo path in the hybrid.

Since the digital echo canceller operates in the sampled data domain, the reccived signal $r(t)$ is sampled at a rate $N / T$ before proceeding the echo cancellation, where $N / T$ is at least twice the bandwidth of $r(t)$, and typically $N \geq 2$ for bipolar transmission. Using the interleaved scheme [2] and denoting the sample points of any continuous-time iunction $x(t)$ as

$$
x_{i}(n)=x\left(n T+\frac{i}{N} T\right), 0 \leq i<N .
$$

The sampled points of $r(t)$ can be expressed as, $0 \leq i<N$

$$
r_{,}(n)=\sum_{m=0}^{M_{1}-1} l_{i}(m) a(n-m)+\sum_{m=0}^{M_{k}-1} k_{2}(m) b(n-m)+\nu_{2}(n)
$$

In which, we assume that the impulse responses $l(t)$ and $k(t)$ are causal and respectively spread no longer $M_{l} T$ and $M_{k} T$ seconds.

The different-phase samples of the received signal can be treated as the outputs of $N$ independent channels and echo 
paths driven by the far-end and near-end sequences of dala symbols. Thus the EC and transmission model is largely simplified, and some implementational advantages can be obtained(see [3]).

The model for the passband transmission is somewhat different from those of the baseband transmission. The models for transmission channel and echo path are modulated to a carrier frequency, and the complex-value operations are required to process the passband signal. After some arrangements, the echo cancellation and receiving procedure can be operated either in baseband or in passband, and all formulations are similar to Eqn. (1). (The details can be referred in [8])

Using the techniques of adaptive filtering, the echo paths in each phase, $k_{i}(m), 0 \leq i<N$, can be estimated recursively. However, the far-end component will occupy the major part of the echo-cancelled residual signal $y_{i}(n)$ when the estimate $\left\{\hat{k}_{i}(m)\right\}$ is adapted around $\left\{k_{i}(m)\right\}$ in a certain degree. If this residual signal is used to adapt the echo cancellers, the estimation will be severly disturbed and not converge. Hence another adaptive estimator for far-end component will be used to cancel this factor in $y_{i}(n)$, based on the decision-directed scheme given in [1]. Thus the configuration of EC with RSG is arranged and shown in Figure 2.

In this decision-directed scheme, the correctness of estimated far-end data symbols specially needs more attention. In section 3 , some methods will be proposed to provide a reliable decoding.

\subsection{Adaptation Procedure}

Among the variety of adaptive filtering algorithms, LMS is commonly used because of its simplicity. Although there are many sophisticated adaptation algorithms, they don't necessarily offer superiorities in this problem. The reason is that the inputs of the adaptive filters, $\{\hat{a}(n)\}$ and $\{b(n)\}$, have only a few bits of precision. In addition, in the case of spread-spectrum communication system, the spectra of the data symbols should be very broad. Hence the eigenvalue spreads of the input data are very close to unity, and this kind of input data is very helpful for the convergence behavior of the LMS algorithm [4].

Because the adaptations of $\widehat{L}_{i}(n)$ and $\widehat{K}_{i}(n)$ are mutually affected, the convergence behavior of these adaptations are quite complicated. In [1], the stability was analyzed only for a few values of $D$. The larger the value of $D$ is, the more complicated the analysis is.

\subsection{The Problem of Multiple Optimal Values of $\left\{\tilde{l}_{i}(m)\right\}$}

In most PSK and QAM transmissions, the sets of data symbols are usually unchanged after some phase-shifting. That is, there exists one maximum integer $R$ such that the phaseshift of multiples of the angle $2 \pi / R$ on any datia symbol will result in another data symbol in the set. For example, it is easily observed that the $M$-ary PSK has an $R$ with value $M$, and 16-ary QAM has the value 4. Hence, from Eqn. (1), it follows that the optimal value of $\left\{\tilde{l}_{i}(m)\right\}$ is not unique. The values, $\left\{e^{\frac{2 \pi}{R} p} \tilde{l}_{i} m\right\}, 0 \leq p<R$, are all optimal values as long as all original data symbols are phase-shifted by the angle $-\pi / R$, respectively.

Thus, if the initial value of the estimate is not properly chosen to near the true value, the estimate will converge to the value whicli is the product of true value and $e^{j \frac{2 \pi}{R} p}$, for some $p$. If this estimate $\left\{\hat{l}_{i}(m)\right\}$ is used in the receiver, the receiver will errorneously decide the true data symbol $a(n)$ to

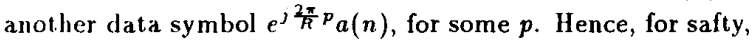
the training period should be added in the first few steps of adaptation. That is, the correct far-end data symbols are used to train the estimate $\left\{\hat{l}_{i}(m)\right\}$ to near the vicinity of the true value.

\section{Reliable Transmission with the Dis- persive Channel}

In a dispersive channel, the dispersion may result in the existence of ISI. Since the information about the dispersive channel is obtained by using the estimate of the dispersed far-end pulse shapes, it is helpful for solving the aforecited problem. In subsection 3.1, we will proposed some demodulation schemes in the receiver by using the effect of dispersion in the channel. In subsection 3.2, the information of the dispersed pulse will be used to develop a Kalman equalized receiver.

\subsection{Optimal Receiving using the Effects of Dis-} persion in Channel

From the thicory of optimal receiver, it is known that the received signal at each baud period can be demodulated by filtering with the matched filter $s^{*}(-t)$ if the transmitted pulse shapes are not severly dispersed. Due to the dispersion in the transmission channel, the original pulse shape is changed to $(1 t)$. llowever, to achieve an optimal demodulator, the matched filter should be designed for this received pulse shape, which includes the effects of dispersion of the channel. Using the matched filter $l^{*}(-t)$, the output will provide a sufficient statistic for decoding the sequence of far-end data symbols [5]. Fortunately, the information about dispersion in channel is included in the estimates $\widehat{L}_{i}(n), 0 \leq i<N$. Hence, the interleaved structure of receiver filter can be proposed and as is shown in Figure 3. In which, $g(n)$ is the output of receiver filter.

Using the maximum-likelihood principle, the far-end data symbol at the $n$-th baud period can be optimally estimated by choosing the data symbol $\hat{a}(n)$ so as to minimize the squared Euclidean distance $|g(n)-\widehat{a}(n)|^{2}$ if no ISI is present, where $g(n)$ is assumed to be normalized w.r.t. the energy of signal pulse $l(1)$.

However, the ISI may present in the dispersed filter output pulse shape $l(t) * l^{*}(-t)$. Hence, some schemes can now be proposed for decoding the output of receiver filter in the presence of ISI.

\section{a. Decision-Feodback Decoding}

From the model decribed in subsection 2, The far-end data symbols seculuence caul be expressed as

$$
n(n)=\left[g(n)-\sum_{m=1}^{M_{1}-1} \Delta_{m}(n) a(n-m)+u(n)\right] / \Delta_{0}(n) .
$$

whice $\Delta_{m}=\sum_{l=0}^{N-1} \sum_{j=0}^{M_{l}-1} l_{i}^{*}(j) l_{i}(j+m)$, and $u(n)$ is the receiver filtering result of echo residue and independent of the far-end data symbols.

Hence, we propose a decision-feedback decoding scheme. (The detailed structure is shown in [8]) In which, $\widehat{\Delta}_{i}(n), 0 \leq$ $i<M_{l}$, are calculated by using the estimates $\hat{l}_{i}(n), 0 \leq i<$ 
$N$, and the far-end data symbols can be estimated by using the optimal decoding method described previously.

It is worthy to noticed that the error propagation in data symbol estimates is dangerous in this decision. Hence other methods may be used to obtain lower bit error probabilities.

\section{b. Trellis Decoding}

Due to intersymbol interference, the data symbols preceding the $\boldsymbol{n}$-th one have some contribution to the value of output of receiver filter, $g(n)$. Here, the decoding trellis for the case of $M_{l}=2$ and binary data $\{ \pm 1\}$ is proposed and shown in Figure $4(a)$. In which, $\widehat{\Delta}_{2}(n)$ is assumed to be tiny and negligible, and the branch cost is defined by using the Euclidean distance. When the trellis is searched to find the minimum'istance estimates of sequences, the effecient Viterbi algorithm can be applied [6]. Figure 4(b) shows the trellis with complex-valued branches for the case of QPSK transmission with $M_{l}=2$ and data symbols $\{ \pm 1 \pm j\}$, where $j=\sqrt{-1}$. In which, the transition from $\alpha$ to $\beta$ is $\alpha \widehat{\Delta}_{0}(n)+\beta \widehat{\Delta}_{1}(n)$. The complexity of trellis increases rapidly as the complexity of the signal constellation and the value of $M_{l}$ grow.

\subsection{Kalman Equalized Receiver using the Infor-} mation about the Dispersed Pulse

Since the channel is disturbed by dispersion and additive noise, the equalizer is used to compensate the effect of dispersion and smooth the noise in the channel. Conventionally, the equalization and the EC worked independently. In the linear equalization, the filter is adapted by using the training or decision-directed schemes without influence on and the requirement of EC. However, in the configuration of EC with RSG, the effect of dispersion in channel is included in the estimate of far-end pulse shape. This information can be used to equalize the channel. Hence a Kalman equalized receiver is proposed, which has the output of far-end data-symbol sequence but not the equalized pulse-shape signal.

\subsubsection{State-Space Reformulation}

If we assume that the echo part is sucessfully cancelled, the output of echo cancellation at the $\boldsymbol{n}$-th baud period can be written, from Eqn. (1), as

$$
\mathbf{Y}(n)=\mathbf{L A}(n)+\eta(n) .
$$

$$
\text { where } \begin{aligned}
\mathrm{Y}(n) & =\left(y_{0}(n), y_{1}(n), \ldots, y_{N-1}(n)\right)^{T}, \\
\mathbf{A}(n) & =\left(a(n), a(n-1), \ldots, a\left(n-M_{l}+1\right)\right)^{T} .
\end{aligned}
$$

$\mathbf{L}$ is a $N \times M_{l}$ matrice with element $\left[l_{i}(j)\right] 0 \leq i<N, 0 \leq$ $j<M_{l}$, and $\eta(n)=\left(\nu_{0}(n), \nu_{1}(n), \ldots, \nu_{N-1}(n)\right)^{T}$.

The data vector $\mathbf{A}(n)$ can be recursively expressed as

$$
\mathbf{A}(n)=\mathbf{M A}(n-1)+(a(n), 0, \ldots, 0)^{T}
$$

where $\mathbf{M}=\left(\begin{array}{c|c}\mathbf{0}_{M_{1}-1}^{T} & 0 \\ \hline \mathbf{I}_{M_{1}-1} & \mathbf{0}_{M_{l}-1}\end{array}\right)$

Applying Kalman filter theory [7] and using the estimate $\hat{\mathbf{L}}(n)$ at the $n$-th baud period, we can obtain the estimate of far-end data-symbol vector as follows:

$$
\begin{aligned}
\mathbf{P}(n)= & \mathbf{M}[\mathbf{P}(n-1)-\mathbf{G}(n-1) \widehat{\mathbf{L}}(n-1) \mathbf{P}(n-1)] \mathbf{M}^{H} \\
& +\mathbf{R}_{a}(n) \\
\mathbf{G}(n)= & \mathbf{P}(n) \widehat{\mathbf{L}}^{H}(n)\left[\widehat{\mathbf{L}}(n) \mathbf{P}(n) \widehat{\mathbf{L}}^{H}(n)+\mathbf{R}_{\nu}(n)\right]^{-1}
\end{aligned}
$$

$z(n)=\mathbf{Y}(n)-\widehat{\mathbf{L}}(n) \mathbf{M A}(n-1 \mid n-1)$.

$\widehat{\mathbf{A}}(n \mid n)=\mathbf{M} \widehat{\mathbf{A}}(n-1 \mid n-1)+\mathbf{G}(n) z(n)$.

where $\mathbf{R}_{\nu}(n)=\sigma_{\nu}^{2}(n) \mathbf{I}, \mathbf{R}_{a}(n)=\left(\begin{array}{c|c}\sigma_{a}^{2}(n) & \mathbf{0}_{N-1}^{T} \\ \hline 0_{N-1} & \mathbf{O}_{(N-1) \times(N-1)}\end{array}\right)$, and $\sigma_{a}^{2}(n)=E\left[|a(n)|^{2}\right] . \widehat{\mathbf{A}}(i \mid j)$ is the eatimate of $\mathbf{A}(i)$ given data from the 0 -th to the $j$-th baud periods.

The equalized receiver has the output of estimated values of far-end data symbols in the signal constellation. These outputs can directly fed to the minimum-distance decision procedure to find the optimal estimates of data symbols in the maximum-likelihood criterion.

From the Kalman equations, (8), (9), and (10), the equalized receiver seems to have a high computational complexity when the value of $N$ is large. However, in some practical applications, the value 2 or a little more, is sufficient for the selection of $N$. Hence the computational complexity can be afforded. If the noise power is very small in the channel (that is. the signal to noise power ratio in the channel is very high), the Kalman estimator can further be simplified as

$$
\widehat{\mathbf{A}}(n)=\hat{\mathrm{L}}^{+}(n) \mathrm{Y}(n) \text {. }
$$

where $\hat{\mathrm{L}}^{+}(n)$ is the pseudoinverse of the matrix $\widehat{\mathrm{L}}(n)$. This simplified equalized receiver can be easily implemented by sone eigen decomposition methods and work well when the signal-to-noise ratio is not too low.

\section{Computer Simulation}

In the following computer simulations, the EC with reliable revceiving methods is performed on two kinds of transmission schemes including QPSK, and 16-ary QAM. The carrier frequency and the baud rate are respectively chosen to be $1800 \mathrm{~Hz}$ and $2400 \mathrm{~Hz}$ which are the same as those of V.32 modem. In these experiments, the durations of pulse-channel response $l(t)$ and pulse-echo path response $k(t)$ spread $2 T$ and $4 T$ seconds, respectively (ie. $M_{l}=2$ and $M_{k}=4$ ), and the value of $N$ is selected to be 8 . Typically, the attenuation in cliannel is $40 \mathrm{~dB}$, and the loss in the hybrid circuits is about $10 \mathrm{~dB}$. Hence the ratio of near-end to far-end signal power $\left(|k|^{2} /|l|^{2}\right)$ is assigned to be $30 \mathrm{~dB}$ in the simulation. Fillally, the additive Gallssian white noise is added in the channel, and make the signal-to-noise ratio equal to $33 \mathrm{~dB}$.

Figure 5 shows the convergence behaviors of estimation errors in the EC system with RSG and the reliable receiving schemes using the effect of dispersion in channel. Observing Figure 5. Hiere are two stages of EC in the direct adaptation sclieme. In the first stage, the main cancellation is for the near-end ecloo signal, and the adaptive RSG cannot be well-adlapted at this time. Due to the errors in the decision of far-end data symbols and in the adaptive RSG, there is more disturbance in the estimation error $\epsilon(t)$, and hence the convergence rate of the adaptive echo canceller is slow. Until the part of near-end echo is cancelled to a certain degree, the adaptation of RSG can work well. Then the far-end signal can be cancelled and the ratio of residual signal to far-end signal power can reach about $-33 \mathrm{~dB}$.

Since the RSG introduces some disturbance to the adaptation of the echo canceller in the early stage of adaptation, the two-stage adaptation scheme is adopted. From Figure 5, the EC of the two-stage adaptation scheme seem converge faster.

If $s^{*}(-t) c^{j 2 \pi f_{c} t}$ is used as the receiver filter, the output valıes of the filter would be disturbed and hence often not 
be decided to the correct far-end data symbols. Figure 6(a) and 7 (a) show the signal constellations of the receiver filter output without using any proposed optimal receiving scheme: and equalization. Since the estimated values of far-end data symbols are always incorrect, the far-end RSG can't correctly produce the counterpart of far-end signal, and hence the echo canceller can not be well-adapted to cancel the echo components. Thus, the resultant signal constellation is chaotic. Figure $6(\mathrm{~b})$ and $7(\mathrm{~b})$ show the signal constellations of the receiver filter output without any equalization, and the echor component is assumed to be perfectly cancelled. (ie. Thif disturbance is resulted only from the effect of dispersion in: channel.) Figure 6(c) and 7 (c) show the signal constell: tions of the filter output for the proposed EC with RSG and equalized receiver after the estimates are converged. It is obviously that the equalized signal points are precisely focused on the points of original sending signal.

\section{Conclusion}

For the EC in data transmission, the double-talk problem can be overcome by using the far-end RSG. However, the transmission channel is usually dispersive. The ISI problem may effect the correctness of the data symbol decision. In this paper, the information in of the far-end RSG is fully used 1.0 deal with this problem and the reliable receiving scliemes are provided. By the computer simulation, the performance and the benefit of the propoesd schemes are shown.

\section{References}

[1] D. D. Falconer, "Adaptive Reference Echo Cantcellation," IEEE Trans. Comm., voi. COM-30, pp.2(183-2094. 1982.

[2] D. G. Messerschmitt, "Echo Cancellation in Speech and Data Transmission," IEEE J. on Sel. Area in Comm., vol. SAC-2, pp.283-297, 1984.

[3] E. A. Lee, and D. G. Messerschmitt, "Digital Communiations," Kluwer Academic Publishers, 1988.

[4] B. Widrow et al., "Stationary and nonstationary learning characteristics of the LMS adaptive filter," Proc. IEEE, vol. 64, pp.1151-1162, 1976.

[5] R. E. Blahut, "Digital Transmission of Information." Addison-Wesley Publishers, 1990.

[6] G. D. Forney, Jr. "The Viterbi Algorithm," Proc. IEIF/ vol. 61, pp.268-278, 1973.

[7] R. E. Kalman, and R. S. Bucy, "New results in linear filtering and prediction theory," Trans. ASME, J. of Basic Eng., vol. 8, pp.95-108, 1961.

[8] H. F. Chi, "The Techniques of Adaptive Filtering and Their Applications in Eclio Cancellation," M.S. Dissestation, National Taiwan University, Taipei, Taiwan R.O.C., 1993.

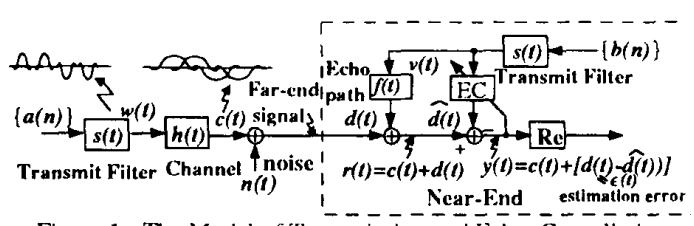

Figure 1 : The Model of Transmission and Iecho Canceltation

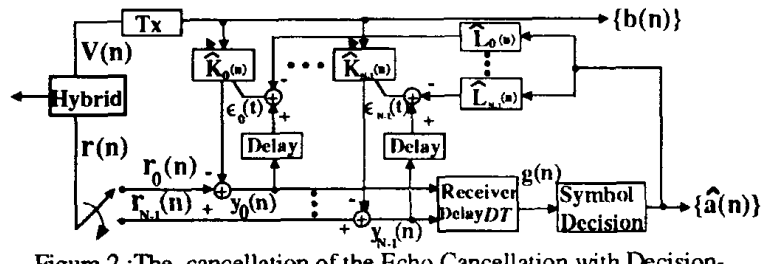

Figure 2 :The cancellation of the Fcho Cancellation with Decision-irected Far-end RSG (where DT is the delay in the receiver)

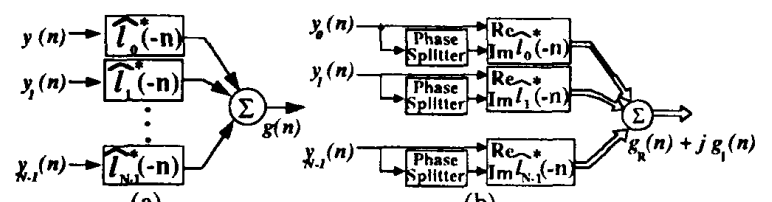

(a)

(b)

Figure 3: The Configurations of the Interleaved Receiver Filtering.

(a) Operation in Baseband. (b) Operation in Passband.

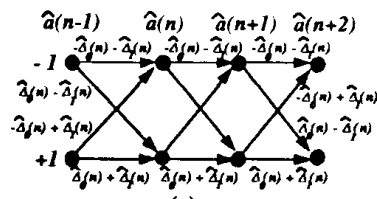

(a)

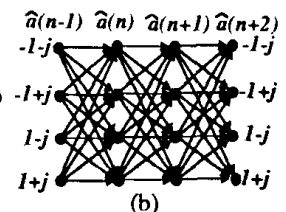

(b)
Figure 4: The Trellis for InterSymbol Interference with $M_{l}=2$. (a) For BJipolar Transmission. (b) For QPSK Transmission

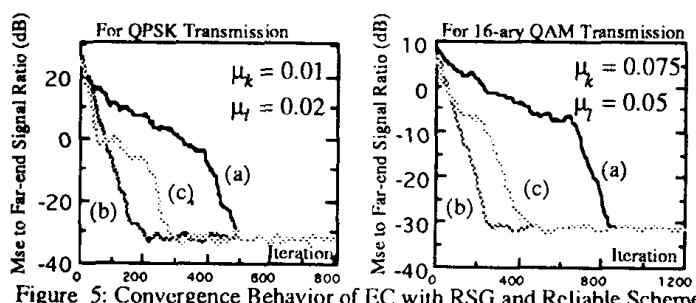

Figure 5: Convergence Behavior of EC with RSG and Reliable Scheme in QPSK and 16-ary QAM Transmission. (a) Direct adaptation. (b) $\wedge$ daptation is allowed to he adapted after 5 it iterations.)

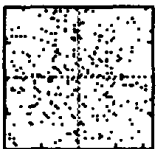

(a)

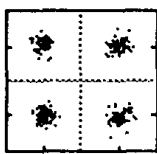

(b)

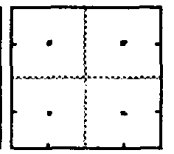

(c)
Figure 6: Signal Constcllation of QPSK (a) Without using any proposed Optimal Receiving scheme and Equalization. (b) Not Equalized and No Echo Component. (distributed only by channel dispersion) (c) Fyualized in the proposed EC system.
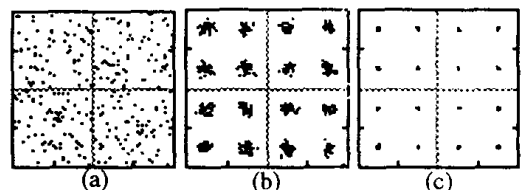

Figure 7: Signal Constellation of 16-ary QAM. (a) Without using any ptoposed Optimal Receiving scheme and Equalization. (b) Not Equalized and No Fcho Component . (disturbed only by channel dispersion) (c) Fqualized in proposed EC Systcm. 